schießende Wirkung aufweist, die eine Vertragsverletzung darstellt, die nur der Gesetzgeber beseitigen kann. Bis auf weiteres ist die Vorschrift europarechtskonform, also vogelschutzrichtlinienkonform in dem Sinne auszulegen, dass nicht beliebige öffentliche Interessen den Tatbestand zu erfüllen imstande sind, sondern von der Art her nur diejenigen, die die Vogelschutzrichtlinie in Art. 9 Abs. 1 V-RL zulässt, und von der Intensität her als ,,zwingend“ zu bewerten sind, sich also als im strengen Sinn erforderliche Maßnahmen erweisen müssen; die Gründe dürfen daher nicht nur unspezifischer sozialer und wirtschaftlicher Art sein ( $\$ 45$ Abs. 7 S. 1 Nr. 5 BNatSchG), sondern sie müssen den Tatbeständen der V-RL entsprechen. Das wäre mit Hilfe einer richtlinienkonformen Reduktion des BNatSchG zu erreichen.

Open Access. Dieser Artikel wird unter der Creative Commons Namensnennung 4.0 International Lizenz veröffentlicht, welche die
Nutzung, Vervielfältigung, Bearbeitung, Verbreitung und Wiedergabe in jeglichem Medium und Format erlaubt, sofern Sie den/die ursprünglichen Autor(en) und die Quelle ordnungsgemäß nennen, einen Link zur Creative Commons Lizenz beifügen und angeben, ob Änderungen vorgenommen wurden.

Die in diesem Artikel enthaltenen Bilder und sonstiges Drittmaterial unterliegen ebenfalls der genannten Creative Commons Lizenz, sofern sich aus der Abbildungslegende nichts anderes ergibt. Sofern das betreffende Material nicht unter der genannten Creative Commons Lizenz steht und die betreffende Handlung nicht nach gesetzlichen Vorschriften erlaubt ist, ist für die oben aufgeführten Weiterverwendungen des Materials die Einwilligung des jeweiligen Rechteinhabers einzuholen.

Weitere Details zur Lizenz entnehmen Sie bitte der Lizenzinformation auf http://creativecommons.org/licenses/by/4.0/deed.de.

Open Access funding enabled and organized by Projekt DEAL.

\title{
https://doi.org/10.1007/s10357-021-3825-3 \\ Ein Landwirtschaftsgesetz für Deutschland im Zeichen von Umweltschutz und Biodiversität - Notwendigkeit, Funktion und Leitbild
}

\author{
Detlef Czybulka/Peter Fischer-Hüftle/Ulrich Hampicke/Wolfgang Köck/José Martinez
}

Der Deutsche Naturschutzrechtstag (DNRT) hat 2018 in seiner „Leipziger Erklärung“ gefordert, ein nationales Landwirtschaftsgesetz mit Betreiberpflichten für die Betriebe zu schaffen, mit dem der dramatische Rückgang der biologischen Vielfalt in der Agrarlandschaft gestoppt werden soll. Gegen diese Stoßrichtung sind Einwände erhoben worden, u. a. im Hinblick auf europäische Zuständigkeiten und begrenzte Bundesgesetzgebungskompetenzen. Die Autoren möchten aufzeigen, dass das Nebeneinander von europäischem und deutschem Landwirtschaftsrecht im Zeichen der Biodiversität, des Umwelt- und Tierschutzrechts neu geordnet werden kann und muss. Dabei ist der deutsche Gesetzgeber in der Verantwortung und hat für die hier entworfene Konzeption auch die erforderlichen Kompetenzen. Der Beitrag besteht aus zwei Teilen.

Prof. Dr. Detlef Czybulka, Universität Rostock,

Vorsitzender des Deutschen Naturschutzrechtstages e. V., Rostock, Deutschland

Peter Fischer-Hüftle, Vors. Richter am VG a. D., Rechtsanwalt, Regensburg, Deutschland

Prof. Dr. Ulrich Hampicke,

ehemals Lehrstuhl für Landschaftsökonomie, Universität Greifswald, Greifswald, Deutschland

Prof. Dr. Wolfgang Köck, Mitglied des Sachverständigenrates für Umweltfragen (SRU),

Leiter des Departments für Umwelt- und Planungsrecht am Helmholtz-Zentrum für Umweltforschung - UFZ,

Leipzig, Deutschland

Prof. Dr. José Martinez,

Geschäftsführender Direktor des Instituts für Landwirtschaftsrecht der Universität Göttingen,

Mitglied des Wissenschaftlichen Beirats für Agrarpolitik,

Ernährung und gesundheitlichen Verbraucherschutz beim BMEL

sowie Zweiter Vorsitzender

der Deutschen Gesellschaft für Agrarrecht,

Göttingen, Deutschland
Die vorliegende Abhandlung befasst sich mit Notwendigkeit, Funktion und Leitbild eines neuen Landwirtschaftsgesetzes. Der demnächst folgende zweite Aufsatz $z^{1}$ widmet sich den erforderlichen materiellen Regelungen und der Finanzierung.

\section{Warum wir ein Landwirtschaftsgesetz brauchen!}

Die Landwirtschaft soll ,nachhaltig, ökologisch verantwortbar, ökonomisch leistungsfähig und regional verankert“ sein und gerade dadurch auch ,einen Beitrag zur Erhaltung lebenswerter ländlicher Räume" leisten, heißt es im (politischen) Leitbild der Bundesregierung für eine zukunftsfähige Landwirtschaft. ${ }^{2}$ Davon ist der Zustand der Landwirtschaft weit entfernt, ganz besonders, wenn auf die ,ökologische Verantwortbarkeit" geschaut wird. Die auf der Hälfte der Fläche Deutschlands ausgeübte industrialisierte und intensive Landwirtschaft ist für den Biodiversitätsverslust in der Kulturlandschaft an erster Stelle verantwortlich. ${ }^{3}$ Der Prozess hat vor mindestens 40 Jahren eingesetzt und beschleunigt sich. Hunderte ,gewöhnliche“ Pflanzenarten (nicht etwa Orchideen), die die Agrarlandschaft seit jeher geprägt hatten, sind auf wenig mehr als fünf Prozent ihrer Population der 1950er Jahre reduziert. ${ }^{4}$

1) Er trägt den Titel „Ein Landwirtschaftsgesetz für Deutschland im Zeichen des Umweltschutzes und der Biodiversität - Zentrale Gesetzesinhalte und Finanzierung" und ist gemeint, wenn nachfolgend auf „Teil 2“ verwiesen wird.

2) Agrarpolitischer Bericht der Bundesregierung 2019, S. $12 \mathrm{ff}$.

3) Nationale Akademie der Wissenschaften (Leopoldina), Artenrückgang in der Agrarlandschaft, Was wissen wir und was können wir tun, 2018

4) Leuschner/Krause/Meyer/Bartels, Strukturwandel im Acker und Grünland Niedersachsens und Schleswig-Holsteins seit 1950. Natur und Landschaft 89, 2014, 386. Weiteres Material u. a. in BfN (Bundesamt für Naturschutz) 2016: Daten zur Natur 2016.; BfN 2017 Agrar-Report 2017, Biologische Vielfalt in der Agrarlandschaft. 
Eine drastische Verringerung der Biodiversität, Verschlechterung der Gewässerqualität und Verarmung des Landschaftsbildes sind Kennzeichen ,moderner" Landwirtschaft, ihre Klimabilanz ist schlecht, und das Tierwohl wird durch Züchtung, Haltungs-, Schlachtungs- und Transportbedingungen massiv geschädigt.

Seit 1994 erteilt Art. 20a GG den Auftrag an die staatlichen Institutionen, die natürlichen Lebensgrundlagen (und seit 2002 auch die Tiere) zu schützen. Ein Staatsziel verwirklicht sich nicht von allein, sondern muss gesetzlich konkretisiert werden. Dabei sind zum einen die landwirtschaftlichen Betriebe in den Blick zu nehmen, die sowohl als Verursacher von Biodiversitäts- und Gewässerschäden, als auch als Träger möglicher Verbesserungsmaßnahmen eine zentrale Rolle spielen. Zum anderen sind Institutionen und Verfahren bereitzustellen, um Maßnahmen zugunsten der Biodiversität zu ermöglichen.

Gesetze mit Steuerungswirkung für die landwirtschaftliche (Boden-)Nutzung sucht man allerdings vergebens. Deutschland hat zwar seit 1955 der Bezeichnung nach ein Landwirtschaftsgeset $z,{ }^{5}$ dieses steuert aber weder die Politik noch die Landwirte. Es zielt auf Einkommenssicherung, postuliert Ernährungssicherheit, Produktivitätssteigerung und den Ausgleich einer vermeintlichen Benachteiligung gegenüber ,,vergleichbaren“ Berufsgruppen und Wirtschaftsbereichen, trägt aber zur Bewältigung der aktuellen Probleme nichts bei.

Dieses Vakuum wird auch nicht dadurch ausgefüllt, dass die Funktionen der Landwirtschaft für das Gemeinwohl, die Erwartungen der Gesellschaft an sie und die sich daraus ergebenden Anforderungen auf andere Weise in ein vom dazu berufenen Gesetzgeber beschlossenes Konzept gegossen wären. Die Anforderungen der guten fachlichen Praxis in $\$ 5$ BNatSchG sind wegen ihrer zu unbestimmten Ausgestaltung weitgehend wirkungslos geblieben. ${ }^{7}$ Europarechtliche Vorgaben (etwa bei der Düngung) werden bisher nicht ausreichend umgesetzt. ${ }^{8}$ Landwirtschaftsbezogene Regelungen im Wasser- und Bodenschutzrecht und über die Pestizidanwendung haben es bislang nicht vermocht, eine umweltverträgliche und biodiversitätserhaltende Landwirtschaft sicherzustellen, ebenso wenig die Bedingungen für Agrarbeihilfen. Solche Bestimmungen sind kein konsistentes Regelwerk, sondern punktuelle Ergebnisse einer Gesetzgebung, die sich zu nicht mehr als dem Allernötigsten entschließen kann. Diese „Flucht vor der Konkretisierung“ hat im Ergebnis zu einer , dauerhaften Abwehrhaltung [der Betriebe] gegenüber umweltrechtlichen Vorgaben" geführt. ${ }^{9}$

Begründet wird diese gesetzgeberische Zurückhaltung u.a. damit, dass die Landwirtschaft wesentlich durch die unionale Agrarpolitik bestimmt werde. ${ }^{10}$ Das hat aber andere Mitgliedstaaten nicht daran gehindert, unter Beachtung der unionalen Vorgaben eigenständige Regelungen zu treffen. Die gegenwärtigen Verhandlungen zur Reform der GAP lassen zudem darauf schließen, dass der Spielraum für eine nationale Agrarpolitik in der EU wieder wachsen wird. Von der deutschen Politik wird zwar zu Recht erwartet, dass sie auf die GAP und die Weiterentwicklung des unionalen Agrarumweltrechts Einfluss nimmt. Ebenso wird aber auch erwartet, dass der Gesetzgeber eine umsetzungsgeeignete Konzeption für die Landwirtschaft erarbeitet und dabei der Beachtung der ökologischen Belastungsgrenzen besondere Aufmerksamkeit entgegenbringt. Dies erfordert eine Neuordnung in einem einheitlichen Gesetzeswerk.

Der Umgang mit den Spielräumen, die eine ökologisierte GAP den Mitgliedstaaten belässt, unterliegt dem Parlamentsvorbehalt; es ist schon deshalb eine wesentliche Entscheidung, weil sie nahezu 50 Prozent der Flächennutzung unseres Landes betrifft und enorme Auswirkungen auf Umweltgüter und Biodiversität hat. Das Staatsziel des Art. 20a GG ist seinerzeit etabliert worden, um dem Schutz der natürlichen Lebensgrundlagen eine gleichrangige verfassungsrechtliche Position wie dem herkömmli- chen Grundrechtsschutz zu verschaffen. ${ }^{11}$ Daher sind Politiken von großer Umweltreichweite, wie die Agrarpolitik, vergleichbar mit grundrechtsrelevanten Eingriffen und müssen auch nach traditionellem Verständnis auf gesetzlicher Grundlage erfolgen. Eine solche Agrarpolitik darf nicht in einem Arkanum von technokratischen Programmen versteckt, sondern muss vom demokratischen Gesetzgeber verantwortet werden, wie es mittlerweile auch für den Klimaschutz anerkannt ist. ${ }^{12}$ Eine Erklärung, aber keine Rechtfertigung für die Untätigkeit des Gesetzgebers mag darin liegen, dass er den Konflikt mit der organisierten Agrarwirtschaft ${ }^{13}$ scheut, die gegen jegliche Reglementierung stets heftigen Widerstand leistet. Als Reaktion darauf kommt es zu Initiativen der Volksgesetzgebung, ${ }^{14}$ die zwar die legislative Lähmung partiell beenden, aber nur ultima ratio sein können, weil Regelungen des Verhältnisses Landwirtschaft und Umwelt bzw. Biodiversität komplex und finanzwirksam sind ${ }^{15}$ und die Landeskompetenz allein dazu nicht ausreicht.

Mit der Leipziger Erklärung hat der 13. DNRT auf die Defizite des Agrarumweltrechts hingewiesen und Reformen gefordert, die angesichts der negativen Umweltauswirkungen der Landwirtschaft und der dramatischen Biodiversitätsverluste von großer Dringlichkeit sind. Nachdem der Ruf nach einem substanziellen Landwirtschaftsgesetz in dieser Zeitschrift ${ }^{16}$ als zur Problemlösung ,wenig geeignet" sowie als nicht kompetenzgerecht zurückgewiesen worden ist, möchten die Autoren aufzeigen, dass sich ein solches Gesetzgebungsprojekt in der Sache lohnt und die Bundesrepublik Deutschland, insbesondere der Bund, auch über die nötigen Gesetzgebungskompetenzen verfügt. Es geht uns um essentielle Grundzüge eines nationalen Landwirtschaftsgesetzes. Grundlegend erscheinen uns Regelungen zur Funktionsbestimmung der Landwirtschaft, insbesondere ein zeitgemäßes Leitbild, Ziele, Schlüsselbegriffe und Instrumente, wobei - in unmittelbarem Zusammenhang mit der GAP - auch der nationale Förderrahmen zur Gewährleistung einer zukunftsfähigen Landwirtschaft eine bedeutende Rolle spielen muss. Von zentraler Bedeutung sind dabei konkrete, unmittelbar geltende, umweltbezogene Grundpflichten der Landwirte, die eine Erhaltung und Wiederherstellung der Vielfalt in der Kulturlandschaft und eine Schonung der Umweltressourcen gewährleisten sollen.

Zwar wird für eine zukunftsorientierte und ,ökologisch verantwortbare" Landwirtschaft auch künftig die ökologische Weiterentwicklung der unionalen GAP von großer Bedeu-

5) Siehe das Landwirtschaftsgesetz v. 5.9.1955, BGBl. I S. 565.

6) Siehe unten zu 4.1

7) Siehe statt vieler: Köck, in: Czybulka/Köck (Hrsg.), Landwirtschaft und Naturschutzrecht, 2019, S. $189 \mathrm{fff}$.

8) Siehe dazu die Verurteilung durch EuGH, Urt. v. 21.6.2018 C 543/16; auch die Düngeverordnung 2017 wird als unzureichend für die Umsetzung der EG-Nitratrichtlinie angesehen, siehe $M \ddot{o}$ ckel, NVwZ 2018, 1599; Douhaire, ZUR 2018, 464. 2020 mussten Düngegesetz und -verordnung ein weiteres Mal novelliert werden, um einer erneuten Verurteilung durch den EuGH zu entgehen.

9) Martinez, DVBl. 2020, 1186, 1190.

10) Vgl. Busse, NuR 2019, 807, 812 f.

11) Vgl. statt vieler: Heselhaus, in: Rehbinder/Schink (Hrsg.), Grundzüge des Umweltrechts, 5. Aufl. 2018, Rdnr. 1.

12) Bundes-Klimaschutzgesetz (KSG) v. 12.12.2019, BGBl. I S. 2513.

13) $\mathrm{Zu}$ den Verflechtungen und Interessen des Deutschen Bauernverbandes (DBV), Studie des iaw-Instituts Arbeit und Wirtschaft, Universität Bremen, im Auftrag des NABU, 64 Seiten, April 2019, Stand 20.2.2021, abrufbar unter https://www. nabu.de/imperia/md/content/nabude/landwirtschaft/agrarreform/190429-studie-agrarlobby-iaw.pdf.

14) Hier ist insbesondere auf das Bayerische Volksbegehren Artenvielfalt „Rettet die Bienen“ aus dem Jahre 2019 hinzuweisen.

15) Martinez, DVB1. 2020, 1186, 1190.

16) Busse, NuR 2019, 807 . 
tung sein. ${ }^{17}$ Doch wird ein nationales Landwirtschaftsgesetz dadurch nicht überflüssig, sondern im Gegenteil als notwendige Ergänzung dringend gebraucht, weil Landwirtschaft und Gesellschaft erwarten dürfen, dass der Staat seine Politik an klaren Grundsätzen ausrichtet und diese rechtlich verankert. Da die Agrarpolitik bislang wesentlich auf der unionalen Ebene gestaltet worden ist, beginnen wir mit einer Analyse der Regelungskompetenzen und mit der Entwicklung der europäischen Agrarpolitik seit 1990 sowie der umweltrechtlichen Einwirkungen auf diese Politik.

\section{Kompetenzgrundlagen für ein Landwirtschaftsgesetz im Spannungsfeld von EU-Recht und deutschem Verfassungsrecht}

Die Agrarpolitik $^{18}$ war bis Ende 2009 eine ausschließliche Zuständigkeit der EU. Seit dem Inkrafttreten des Lissabonner Vertrags im Dezember 2009 gehört sie aber genauso wie die Umweltpolitik zu den sog. geteilten Zuständigkeiten, d.h. sowohl die Union als auch die Mitgliedstaaten dürfen diese Politikbereiche regeln (Art. 2 Abs. 2 S. 1 AEUV). Soweit die Union ihre Zuständigkeit nicht ausgeübt hat, dürfen die Mitgliedstaaten ihre Zuständigkeit wahrnehmen (Art. 2 Abs. 2 S. 2 AEUV). Dies entspricht der konkurrierenden Gesetzgebungsbefugnis im nationalen Verfassungsrecht. ${ }^{19}$ Eine Sperrwirkung für die Mitgliedstaaten ergibt sich daher erst dann, wenn Unionsrecht keinen Raum für weitere substanzielle Regelungen lässt. ${ }^{20}$ Bei agrarrechtlichen Regelungen der Mitgliedstaaten ist daher zu untersuchen, ob eine Materie durch die Union erschöpfend geregelt ist. Jenseits dessen ergänzen unionale und nationale Regelungen einander. Mit Blick auf die hier besonders interessierenden Gegenstände eines nationalen Landwirtschaftsgesetzes kann auf eine Sperrwirkung des Unionsrechts nur sehr begrenzt erkannt werden.

Soweit eine unionale Regelung ihren Schwerpunkt nicht im Bereich der Agrarpolitik, sondern in der Umweltpolitik hat, dürfen die Mitgliedstaaten schon wegen Art. 193 AEUV weitergehende Regelungen treffen. ${ }^{21}$ Das ist wichtig auch mit Blick auf ein nationales Landwirtschaftsgesetz, soweit dessen Regelungen den Umweltschutz betreffen. Dem nationalen Gesetzgeber steht es frei, Umweltschutzregelungen in Fachgesetzen des Umweltschutzes oder in einem Landwirtschaftsgesetz zu verorten. ${ }^{22}$ Regelungen des Unionsrechts, die auch dem Tierschutz dienen, wie etwa Regelungen zur Nutztierhaltung ${ }^{23}$, sind demgegenüber keine Umweltvorschriften, sondern unterliegen der Agrarkompeten $\mathrm{z}^{24}$ sowie den Vorschriften über das Funktionieren des Binnenmarkts (Art. 38 Abs. 2 AEUV). Im Übrigen unterliegen alle unionalen Regelungen dem Subsidiaritätsprinzip des Art. 5 Abs. 3 EUV; d.h. die Union darf ihre Kompetenzen überhaupt nur ausüben, soweit die Ziele der in Betracht gezogenen Maßnahmen von den Mitgliedstaaten nicht ausreichend verwirklicht werden können.

Schaut man auf die Gesetzgebungskompetenzen im Grundgesetz, so steht dem Bund ein umfassender Kompetenztitel für die Landwirtschaft nicht zu. ${ }^{25}$ Vielmehr sind landwirtschaftsrelevante Gesetzgebungsmaterien in unterschiedlichen Kompetenztiteln angesprochen. Von besonderer Bedeutung ist Art. 74 Nr. 17 GG, der die Förderung der landwirtschaftlichen Erzeugung, die Sicherung der Ernährung und die Einund Ausfuhr landwirtschaftlicher Erzeugnisse umfasst. Darüber hinaus finden sich landwirtschaftsrelevante Materien in Art. 74 Nr. 20 GG (Pflanzenschutz, Tierschutz), Art. 74 Nr. 18 GG (Bodenrecht), Art. 74 Nr. 31 GG (Raumordnung) und Art. 74 Nr. 11 GG (Recht der Wirtschaft). Landwirtschaftsrelevant sind auch viele Umweltschutzmaterien. Hier hat der Bund seit der Föderalismusreform weitgehende Gesetzgebungsrechte in den Bereichen Luftreinhaltung und Klimaschutz (Art. 74 Nr. 24 GG), Gewässerschutz (Art. 74 Nr. 32 GG), Naturschutz (Art. 74 Nr. 29 GG) und Abfallwirtschaft (Art. 74 Nr. 24 GG). Diese Bundeskompetenzen sind teilweise mit Abweichungsrechten der Länder kompensiert worden (Art. 72 Abs. 3 Nrn. 2 und 5 GG), ${ }^{26}$ enthalten aber auch abweichungsfeste Materien (wie z.B. den Artenschutz und stoffbezogene wasserrechtliche Regelungen).

Daraus ergibt sich mit Blick auf ein neues Landwirtschaftsgesetz des Bundes, dass die Teilkompetenzen des Bundes in der Summe umfassende Regelungen ermöglichen und lediglich einige Materien teilweise unter Abweichungsvorbehalt stehen. In der Literatur wird meist auf die Basiskompetenz des Art. 74 Nr. 17 GG abgestellt ${ }^{27}$ und einschränkend darauf hingewiesen, dass dieser Kompetenztitel im Kern lediglich Förderregelungen abdecke. ${ }^{28}$ Demgegenüber hat das BVerfG insoweit auch eingreifende Regelungen anerkannt. ${ }^{29}$ Davon geht auch der Bundesgesetzgeber aus, er hat z. B. das Düngegesetz zur Umsetzung der Nitratrichtlinie maßgeblich auf Art. 74 Nr. 17 GG gestützt. ${ }^{30}$ Bei den Teilmaterien der Sicherung der Ernährung und der Ein- und Ausfuhr landwirtschaftlicher Erzeugnisse ist nie bezweifelt worden, dass die Kompetenzgrundlage auch eingreifende Maßnahmen umfasst. ${ }^{31}$ Dass die Aufnahme der Ernährungssicherung historisch bedingt war und auf die Nachkriegssituation abzielte, ${ }^{32}$ kann nicht dazu führen, ihr heute - beispielsweise unter Hinweis auf den Weltmarkt für Agrarprodukte - jeglichen Anwendungsbereich abzusprechen. Im Gegenteil: die kritischen Anfragen an eine ungehemmte Globalisierung und die Erfahrungen einer zunehmend vulnerablen Welt (Stichworte: Pandemien, Klimawandel, politische Umbrüche) weisen darauf hin, dass die Sicherung der Ernährung nach wie vor im öffentlichen Interesse liegt. ${ }^{33}$ Instrumente dafür finden sich in der GAP, haben dort aber keinen abschließenden Charakter.

Dagegen besteht bei der Ein- und Ausfuhr landwirtschaftlicher Erzeugnisse Unionsrecht auf der Basis der EU-Binnenmarkt- und der EU-Außenhandelskompetenzen. Wegen des Vorrangs des Europarechts werden hier nationale Alleingänge nicht in Betracht kommen, sondern es wird nach gemeinsamen unionalen Strategien zu suchen sein. Dass

17) Dazu auch die gemeinsame Stellungnahme von Leopoldina, Acatech und der Union der deutschen Akademien der Wissenschaft, Biodiversität und Management von Agrarlandschaften, 2020, S. 51

18) Dazu Streinz, EUV/AEUV, 2. Aufl. 2012, zu Art. 4 AEUV, Rdnr. 7; Kloepfer, NuR 2018, 11.

19) Siehe nur Calliess, in: Calliess/Ruffert, EUV/AEUV-Kommentar, 5. Aufl. 2016, zu Art. 2 AEUV, Rdnr. 12 und zu Art. 4 AEUV, Rdnr. 1.

20) Dazu näher Calliess, zu Art. 2 AEUV, Rdnr. 15.

21) Dazu auch Calliess, in: Calliess/Ruffert, zu Art. 2 AEUV, Rdnr. 13

22) Dazu auch Rehbinder, in: Czybulka/Köck (Hrsg.), Landwirtschaft und Naturschutzrecht, 2019, S. 77, 90.

23) Siehe etwa die Richtlinie 2008/119/EG des Rates vom 18.12.2008 über Mindestanforderungen für den Schutz von Kälbern, ABl. 2009 L 10, S. 7.

24) I. V.m. Art. 13 AEUV (Querschnittsklausel Tierschutz). Dazu näher Cymutta, in: Härtel, Handbuch des Fachanwalts Agrarrecht, Kapitel 25, Rdnr. 9-17.

25) A.A. Maunz, in: Maunz/Dürig, GG-Kommentar, 80. EL 2020 , Art. 74 Nr. 17 Rdnr. 195: ,,umfassende Kompetenz für das Recht der Agrarwirtschaft"

26) So dürfen die Länder sowohl im Bereich des Naturschutzes als auch im Bereich des Gewässerschutzes vom Bundesrecht abweichende Regelungen treffen.

27) Siehe etwa Rehbinder, in: Czybulka/Köck, S. 77, 90 ff.; Busse, NuR 2019, 807, $811 \mathrm{f}$.

28) So Busse, NuR 2019, 807, 811

29) BVerfG, Beschl. v. 6.5.2014, NVwZ 2014, 1306 (Weinabgabe); Beschl. v. 23.6.1981, NVwZ 1982, 29 (Auflösung von Wasserund Bodenverbänden)

30) Vgl. BT-Drs. 18/7557, S. 14

31) Vgl. Seiler, in: BeckOK GG, zu Art. 74, Rdnr. 60; Degenhart, in: Sachs (Hrsg.), zu Art. 74, Rdnr. 69.

32) Vgl. Seiler, in: BeckOK GG, zu Art. 74, Rdnr. 60.

33) Dazu Martinez, DVB1. 2020, 1186, 1188 m.w. N. 
die Gewährleistung einer umweltverträglichen Landwirtschaft dazu führen muss, eine „ökologisch verantwortbare“ Agrarpolitik gegenüber Agrarimporten abzusichern, die der Umweltverträglichkeit keine Beachtung schenken, ${ }^{34}$ steht auf einem anderen Blatt und ist durch den „Green Deal" und die ,farm to fork"-Strategie der EU-Kommission in Ansätzen bereits adressiert. ${ }^{35}$

Im Ergebnis ist festzuhalten, dass die Landwirtschaftspolitik sowohl in der Zuständigkeit der EU wie der Mitgliedstaaten liegt und ihre Ökologisierung auf verschiedene umweltbezogene Kompetenztitel gestützt werden kann. Auf der nationalen Ebene ergeben sich aus verschiedenen Kompetenztiteln umfassende Regelungsbefugnisse für den Bund, mit denen das Projekt eines neuen Landwirtschaftsgesetzes verfolgt werden kann.

\section{Entwicklung der europäischen Agrarpolitik und des Agrarumweltrechts, Kompetenzgrundlagen}

\subsection{Ziele der gemeinsamen Agrarpolitik, fehlende Agrarumweltziele}

Gemäß Art. 39 AEUV zielt die gemeinsame Agrarpolitik darauf ab, (a) die Produktivität der Landwirtschaft durch Förderung des technischen Fortschritts, Rationalisierung der landwirtschaftlichen Erzeugung und den bestmöglichen Einsatz der Produktionsfaktoren, insbesondere der Arbeitskräfte zu steigern, (b) auf diese Weise der landwirtschaftlichen Bevölkerung (...) eine angemessene Lebenshaltung zu gewährleisten, (c) die Märkte zu stabilisieren, (d) die Versorgung sicherzustellen und (e) für die Belieferung der Verbraucher zu angemessenen Preisen Sorge zu tragen. Art. 39 Abs. 2 AEUV zählt eine Reihe von Bedingungen auf, die bei der Zielverfolgung zu berücksichtigen sind, der Schutz der Umwelt gehört aber nicht dazu. Erst 1993 ist eine Querschnittsklausel für den Umweltschutz eingefügt (heute Art. 11 AEUV) sowie eine explizite europäische Umweltpolitik (heute Art. $192 \mathrm{ff}$. AEUV) etabliert worden. Umwelterfordernisse waren bis dahin im Wesentlichen durch die Mitgliedstaaten sicherzustellen. ${ }^{36}$

\subsection{Gemeinsame Agrarpolitik (GAP) nach 1990 und Globalisierung}

Eine durch Außenschutz, Mindesterzeugerpreise, Exportsubventionen und staatliche Aufkäufe (,Intervention“) charakterisierte Preispolitik prägte die ersten Jahrzehnte. ${ }^{37} \mathrm{Um}$ 1990 hatte sich dieses System totgelaufen. Die Überproduktion (,Butterberge“) war nicht mehr zu finanzieren, dem Druck von außen gegen die Wettbewerbsverzerrungen zugunsten der eigenen Bauern nicht mehr zu widerstehen. Die sich abzeichnende Erweiterung der EU um Agrarländer wie Polen und Rumänien schaffte zusätzlichen Druck. Auch die weltpolitischen Veränderungen und die deutsche Wiedervereinigung dürften eine Rolle gespielt haben.

Die ,MacSharry-Reform“ von 1992 bestand in der Hauptsache in der Abschaffung der gestützten Preise, vor allem des Getreides, und deren schrittweiser Absenkung und schließlich Freigabe auf das niedrigere Weltmarktniveau. Zur Abfederung und vorübergehenden Gewährleistung des Einkommens der Betriebe entstand ein Prämiensystem, ,Preisausgleichsmaßnahmen“ genannt. Spätere Neuausrichtungen ${ }^{38}$ vereinfachten das Prämiensystem, womit sich der Spielraum für die Betriebe beständig erweiterte, ihre Produktionsrichtungen nach ihrem Ermessen zusammenzustellen (Entkoppelung). Gab es zu Beginn Prämien nur für Flächen, auf denen zuvor preisgestützte Kulturen angebaut wurden (also z. B. nicht für Grünland), so ging die Entwicklung hin zu einer universellen Flächenprämie von derzeit (2020) 250 bis 300 Euro pro Hektar und Jahr, finanziert in Deutschland mit etwa fünf Milliarden Euro pro Jahr. Sie heißt nach 30 Jahren listigerweise nicht mehr „Preisausgleichsmaßnahme“, sondern „Direktzahlung“ im Rahmen der „Ersten Säule“.
Einen Abschluss des Liberalisierungsprozesses bildete um 2015 die Abschaffung der 1984 eingeführten „Garantiemengenregelung "im Sinne einer Kontingentierung bei der Milch. Von nun an konnte jeder so viel Milch erzeugen wie er wollte, allerdings ohne Preisgarantie. Um 2015 konnte man (etwas vereinfacht) sagen, dass es in Deutschland seit 130 Jahren zum ersten Mal keine produktbezogenen Stützungsprogramme mehr gibt. Der einzige direkte politische Einfluss auf die Flächennutzung ist die Förderung des Energiepflanzenanbaus für die Erzeugung von Biogas, Strom, Biodiesel und Bioethanol auf über 2 Millionen Hektar. Neben zahllosen komplizierten und der Öffentlichkeit oft unbekannten Privilegien auch auf nationaler Ebene, wie besonders in der Steuer- und Sozialpolitik, ${ }^{39}$ gibt es mit der Ersten Säule eine an die Fläche gebundene staatliche Pauschalzahlung, deren sachliche Rechtfertigung immer schwieriger wird.

Allerdings gibt es in der Ersten Säule (Europäischer Garantiefonds für die Landwirtschaft EGFL, marktbezogene Ausgaben und Direktzahlungen) nach wie vor Möglichkeiten der Intervention und in einigen Bereichen Elemente einer Mengensteuerung. ${ }^{40}$ Auch die Direktzahlungen sind nicht vollständig von der Produktion entkoppelt. ${ }^{41} 2018$ betrugen die sog. Marktausgaben der EU insgesamt „nur“ 2,7 Milliarden Euro, während sich die Direktzahlungen auf 41,48 Milliarden Euro beliefen, also etwa 93 Prozent der Mittel aus der Ersten Säule.

Seit etwa 2010 schlagen die Effekte der Globalisierung voll durch. Die EU ist kein abgeschlossener Agrarmarkt mehr, sondern ihre Betriebe nehmen direkt am Weltmarkt teil. Das heißt, dass sich auch die Preise vor allem der ,grandes cultures" auf dem Weltmarkt bilden. Einerseits sind diese durch wachsende Nachfrage in Asien gestiegen, was an der Rechtfertigung der Ersten Säule nagt. Andererseits wird der Einfluss der EU auf sie immer geringer. Beim Weizenpreis ist weniger erheblich, wie viel die europäischen Bauern erzeugen. Damit entfällt zumindest hier das jahrzehntelang drückende Problem „Überproduktion und Preisdruck“. Es bedarf keiner die Produktion senkenden Maßnahmen mehr, wie obligatorischer Flächenstilllegungen, die einst erheblichen ökologischen Nutzen stifteten. Im Zusammenhang mit dem Anbau von Energiepflanzen sowie weiteren Intensivierungstendenzen ist damit der Druck auf die Reste der Lebensräume für Tier- und Pflanzenarten im Agrarraum weiter angestiegen.

Die Erwartung, dass mit der Deregulierung die Betriebe zu ihrem (sei es auch eng verstandenen) ökonomi-

34) Siehe dazu die Diskussionszusammenfassung „Landwirtschaft und Umweltschutz“ im Rahmen der GfU-Fachtagung 2019, Rdnr. 2.

35) Vgl. EU-Kommission, COM (2020) 381 final, S. 21.

36) Siehe insoweit schon $\$ 8$ Abs. 7 und $\$ 1$ Abs. 3 BNatSchG 1976.

37) Rutz/Schramek, in: Möckel/Köck (Hrsg.), UFZ-Abschlussbericht „Rechtliche und andere Instrumente für vermehrten Umweltschutz in der Landwirtschaft, November 2013, S. 28.

38) 1999: „Agenda 2000“, 2003: „Halbzeitbewertung“, 2009: Health Check", 2013: GAP-Reform.

39) Für 2019 sind im Haushalt des BMEL insgesamt rund 4 Milliarden Euro für die eigenständige landwirtschaftliche Sozialpolitik veranschlagt (Agrarpolitischer Bericht der Bundesregierung 2019, Teil B, Kap. 8.1, Tabelle 15 bis Tabelle 18). 2010 betrugen die Sollausgaben des Bundes für die landwirtschaftliche Sozialpolitik 3,8 Milliarden Euro, vgl. Rutz/Schramek, in: Möckel/ Köck (Hrsg.), UFZ-Abschlussbericht „Rechtliche und andere Instrumente für vermehrten Umweltschutz in der Landwirtschaft, November 2013, S. 13.

40) Art. 136 VO (EU) Nr. 1308/2013 über eine gemeinsame Marktorganisation für landwirtschaftliche Erzeugnisse und zur Aufhebung der Verordnungen (EWG) Nr. 922/72, (EWG) Nr. 234/79, (EG) Nr. 1037/2001 und (EG) Nr. 1234/2007, sog. Änderungsverordnung und Anhang XIII.

41) Angaben aus Agrarpolitischer Bericht der Bundesregierung 2019, 2020, S. 130 
schen Wohl finden würden, hat sich nur teilweise erfüllt Das Auslaufen der Milchkontingentierung führte dazu, dass zahlreiche Großbetriebe jeweils Hunderte von neuen Kühen einstellten mit dem voraussehbaren Ergebnis einer ernsten Milchpreiskrise im Jahre 2016. Sind auch Butter, Käse und Magermilchpulver durchaus ,globalisierte“ Produkte, so bildet sich der Milchpreis anders als beim Weizen immer noch in geographischen Bezugsräumen.

\subsection{Entwicklung des EU-Agrarumweltrechts}

Eine explizite europäische Umweltpolitik ist vergleichsweise spät etabliert worden. Auch die Einbeziehung umweltbezogener Erfordernisse in die europäische Agrarpolitik gibt es erst seit den 1990 er Jahren. ${ }^{42}$ Bis dahin war der Umweltschutz im Wesentlichen eine ausschließliche Angelegenheit der Mitgliedstaaten. Deutschland hat sich mit umweltbezogenen Anforderungen an die Landwirtschaft allerdings sehr zurückgehalten. Es dominierte eine Politik der Privilegierung. Bis spät in die 1990er Jahre galt die gesetzliche Vermutung, dass eine ordnungsgemäße Landwirtschaft auch den Zielen des Naturschutzes und der Landschaftspflege dient. ${ }^{43}$ Zudem war früh klargestellt, dass das Ausbringen von Düngemitteln und Pestiziden nicht als Gewässerbenutzung zu qualifizieren ist, sodass die wasserwirtschaftlichen Instrumente weitgehend unanwendbar blieben. ${ }^{44}$ Noch heute ist eine Sonderstellung der Landwirtschaft charakteristisch für das nationale Umweltrecht.

Wirksame Umweltanforderungen an die Landwirtschaft sind vor allen Dingen durch die europäische Gesetzgebung etabliert worden, nämlich durch ein Kontrollrecht für Düngemittelprodukte und Pflanzenschutzmittel, ${ }^{45}$ Verpflichtungen zur Begrenzung des Nitrateintrags ${ }^{46}$ und Naturschutzvorschriften für bestimmte Lebensräume und Arten. ${ }^{47}$ Bei der Umsetzung hat sich Deutschland bis 2020 den Verpflichtungen aus der Nitrat-Richtlinie weitgehend entzogen. ${ }^{48}$ Auch die Vogelschutz- und die FFH-R ichtlinie sind über Jahrzehnte nur unzureichend umgesetzt worden. ${ }^{49}$ Zugunsten der Landwirtschaft wird entgegen den tatsächlichen Auswirkungen gesetzlich vermutet, dass eine Bewirtschaftung der Flächen nach guter fachlicher Praxis mit den artenschutzrechtlichen Erfordernissen in Einklang stehe ( $\$ 44$ Abs. 4 BNatSchG). Impulse für mehr Umweltschutz in der Landwirtschaft kamen in der Folgezeit kaum mehr durch das europäische oder nationale Umweltrecht, sondern durch die Gemeinsame Agrarpolitik. Auch die Schaffung eines verbindlichen Rechtsrahmens für den Ökolandbau und seine Produkte beruht auf EU-Recht. ${ }^{50}$

Teilweise nach dem Vorbild der Schweiz wurden ab dem Jahre 2005 die Zahlungen aus der Ersten Säule an die Einhaltung von Verpflichtungen auf den Gebieten Umwelt, Tiergesundheit und Verbraucherschutz gebunden (,Cross Compliance"). Soweit sich die Verpflichtungen auf Verordnungen und Richtlinien der EU beziehen, verlangen sie nichts anderes als die Selbstverständlichkeit, Gesetze einzuhalten. Die darüber hinausgehenden Verpflichtungen ( $a b$ 2015 auch „Greening“) zur Erhaltung der Ackerflächen in einem guten ökologischen und landwirtschaftlichen $\mathrm{Zu}-$ stand, z.B. in Gestalt von Fruchtfolgen mit einer Mindestanzahl von Gliedern, erfordern so wenig zusätzlichen Aufwand, dass sie von den meisten Betrieben von allein eingehalten wurden, weil für das Greening 30 Prozent der Direktzahlungen geleistet wurden. Anspruchsvollere Maßnahmen mit höherem Gewichtungsfaktor, z. B. artenreiche Ackersäume und Pufferstreifen mit einem Gewichtungsfaktor von 1,5 wählte trotz dieser hohen Gewichtung fast niemand als ,ökologische Vorrangflächen“.

Aus heutiger Sicht gilt die Kritik weniger den Maßnahmen des Greenings als solchen als ihrer mangelnden regionalen Bezogenheit und - vor allem - der viel zu großen Wahlmöglichkeiten für den einzelnen Betrieb, sodass das Projekt „ökologischer Vorrangflächen“ auf 5 Prozent der Ackerflächen als gescheitert angesehen werden muss. Das kann man besser und auf viel größerer Fläche mit - absolut gesehen - gleicher Förderungshöhe machen. ${ }^{51}$

Schon in den 1980er Jahren wurde von der Wissenschaft die Notwendigkeit erkannt, der industrialisierten Landwirtschaft ein System ökologischer Förderungen entgegenzustellen, mit regional durchaus bemerkenswerten Erfolgen. ${ }^{52}$ In der MacSharry-Reform wurde hieraus eine der „flankierenden Maßnahmen“. Die Mitgliedstaaten durften Landnutzungen, die Umweltressourcen wie Böden und Gewässer sowie die Biodiversität schonten, mit Agrarumweltprogrammen und dem Vertragsnaturschutz fördern. Anfangs war zu kritisieren, dass angesichts sehr geringer Anforderungen tatsächlich Subventionen im ,grünen Mäntelchen“ erfolgten, zumal solche Zahlungen ausdrücklich auch eine Marktentlastungs- und eine Einkommensfunktion haben sollten. Von der „Agenda 2000“53 ab durften die Zahlungen nur noch mit ökologischen Zielen erfolgen.

Seit 2005 greift als wichtigstes Element der „Zweiten Säule" die ELER-Verordnung. ${ }^{54}$ Während der Förderperiode 2007-2014 ${ }^{55}$ bestanden in recht übersichtlicher Weise vier Schwerpunkte (,axes“): Wettbewerbsfähigkeit, Umweltschutz, Verbesserung der Lebensqualität und LEADER. ${ }^{56}$ Die deutschen Länder hatten weitgehende Freiheiten bei der Bedienung dieser ,axes“, wobei Bayern, Baden-Württemberg und Nordrhein-Westfalen über 50 Prozent der Mittel für den Umwelt- und Naturschutz einsetzten, sich dagegen andere, wie besonders Niedersachsen und Hamburg, im Bereich der geforderten Untergrenze von 25 Prozent bewegten. Für die Förderperiode 20142020 greift die VO (EU) 1305/13. Die Mittel sind mit etwa 2,4 Milliarden Euro pro Jahr in Deutschland ähnlich hoch wie zuvor, bestehend aus EU-Zuweisungen, Kofinanzierungen des Bundes, freiwilligen „top ups“ (der Länder) und Mitteln aus der „Modulation“" in Höhe von knapp 200 Millionen Euro. Mit der letzteren gestattet die EU den Staaten, bis zu 15 Prozent der Mittel aus der Ersten in die Zweite Säule umzuschichten. Von dieser zu begrüßenden Mög-

42) Siehe oben zu 2

43) Siehe insoweit schon $\$ 8$ Abs. 7 und $\$ 1$ Abs. 3 BNatSchG 1976.

44) Durch die (allerdings finanziell ausgleichspflichtige) Ausweisung von Wasserschutzgebieten ist immerhin für einen wirksamen Trinkwasserschutz Sorge getragen worden. Näher dazu: Köck, in: Czybulka/Köck, Landwirtschaft und Naturschutz, 2019, S. 189, $194 \mathrm{ff}$.

45) Dazu näher: Köck, in: Czybulka/Köck, Landwirtschaft und Naturschutz, 2019, S. 189, $197 \mathrm{f}$.

46) Nitrat-Richtlinie 1991, dazu Köck, in: Czybulka/Köck, Landwirtschaft und Naturschutz, 2019, S. 189, $198 \mathrm{f}$.

47) Vogelschutz-Richtlinie 1979; FFH-Richtlinie 1992

48) Vgl. Köck, in: Czybulka/Köck, Landwirtschaft und Naturschutz, 2019, S. 189, $197 \mathrm{ff}$.

49) Siehe EuGH, Urt. v. 10.1.2006 - C-98/03, NuR 2006, 166 und jüngst die mit Gründen versehene Stellungnahme der Kommission vom 12.2.2020 im Vertragsverletzungsverfahren Nr. 2014/2262, betreffend die Bundesrepublik Deutschland, S. $15 \mathrm{f}$

50) Siehe VO (EWG) Nr. 2092/91 sowie die Weiterentwicklung in der VO (EG) Nr. 834/2007.

51) Siehe Teil 2 zu 4

52) Schumacher, Schutz und Erhaltung gefährdeter Ackerwildkräuter durch Integration von landwirtschaftlicher Nutzung und Naturschutz. Natur und Landschaft 55, 1980, 447-453.

53) Verordnung (EG) Nr. 1257/1999 des Rates vom 17.5.1999 über die Förderung der Entwicklung des ländlichen Raums durch den Europäischen Ausrichtungs- und Garantiefonds für die Landwirtschaft (EAGFL) und zur Änderung bzw. Aufhebung bestimmter Verordnungen, AB1. L 160, S. 80.

54) Europäischer Landwirtschaftsfonds für die Erhaltung des ländlichen Raumes, VO 1698/05.

55) Ausführliches in Hampicke, Kulturlandschaft und Naturschutz, 2013, S. $175 \mathrm{ff}$.

56) Freese, Natur- und Biodiversitätsschutz in ELER. Finanzielle Ausgestaltung der Länderprogramme zur Ländlichen Entwicklung. Naturschutz und Landschaftsplanung 44, 69-76. 
lichkeit, eine bloße Subvention zumindest teilweise in ein Leistungseinkommen zu verwandeln, hat Deutschland nur sehr zurückhaltend Gebrauch gemacht und jahrelang einen Satz von 4,5 Prozent angewendet, der für 2020 auf 6 Prozent erhöht wurde. ${ }^{57}$ Bei ELER gibt es nun sechs Prioritäten, namentlich Wissenstransfer und Innovation, Wettbewerbsfähigkeit der Landwirtşchaft, Förderung der Nahrungsmittelkette, Schutz von Ökosystemen, Klimaschutz und Bekämpfung von Armut. Hier ist eine Schwerpunktbildung für den Schutz und die Wiederherstellung der Biodiversität weniger zu erkennen.

\subsection{Aktuelle Entwicklungen der GAP- zum Bedeutungsgewinn nationaler Landwirtschaftspolitik}

Im Juni 2018 veröffentlichte die EU-Kommission Vorschläge zur neuen GAP nach 2020. ${ }^{58}$ Ende 2020 standen noch nicht alle Rahmenbedingungen fest. Der Beginn der neuen GAP wird daher voraussichtlich um zwei Jahre auf den 1.1.2023 verschoben. In der Agrarumwelt- und Klimaschutzpolitik wird das bisherige Greening-Modell durch Konditionalitäten ersetzt. Dies sind Anforderungen, die jeder Landwirt umsetzen muss, um die Basisprämie zu erhalten. Die bisherigen ökologischen Bedingungen für die Direktzahlungen (Cross-Compliance und Greening) sollen durch zusätzliche zwingende Umweltmaßnahmen ergänzt werden. Dazu gehören der Zwischenfruchtanbau auf der gesamten Ackerfläche oder ein festgelegter Anteil an Brachen.

Ein weiterer Baustein der künftigen Ökokonditionalität der Direktzahlungen sind Öko-Regelungen („Ecoschemes"), d.h. einjährige Agrarumwelt- und Klimaschutzmaßnahmen, die zwingend in jedem Mitgliedstaat vorzusehen sind, deren Umsetzung aber für den Landwirt freiwillig ist. Die Kommission und der Rat haben vorgeschlagen, dass mindestens 20 Prozent (EU-Parlament: 30 Prozent) der Mittel aus der 1. Säule für die „Ecoschemes“ eingesetzt werden. Die Höhe der Zahlungen soll nicht nach den anfallenden Kosten und Aufwendungen, sondern aufgrund des ökologischen Mehrwerts berechnet werden. Beispiele sind verschiedene Streifenmaßnahmen, Spätschnitt im Grünland oder ein vielfältiges Fruchtartenspektrum. Zentrale Bedeutung kann ferner den Möglichkeiten der Umschichtung von Finanzmitteln aus den Direktzahlungen in die zweite Säule zukommen.

Während diese Konditionalitäten das bisherige Greening-Modell fortschreiben, findet ein grundlegender Wandel durch das sog. ,neue Umsetzungsmodell“" statt. Künftig soll die EU nur noch grundlegende Anforderungen für die Umsetzung und Kontrolle festlegen. Jeder Mitgliedstaat ist verpflichtet, der EU einen nationalen Strategieplan vorzulegen, der für beide Säulen gilt. Dort sollen landesweit die Ziele, die Maßnahmengestaltung und die Mittelansätze festgelegt werden, die als detaillierte Regelungen für die landwirtschaftlichen Betriebe gelten. Diese Strategiepläne sollen genutzt werden, um den regionalen naturräumlichen Gegebenheiten und den Präferenzen der Gesellschaft Rechnung zu tragen. Sie müssen mit den übergeordneten Zielen der GAP im Einklang stehen.

Wenn das neue Umsetzungsmodell realisiert wird, wovon mit Sicherheit auszugehen ist, muss die Bundesregierung die grundlegenden Parameter der deutschen Landwirtschaft definieren, die gefördert werden sollen. Diese Definition obliegt, wie bereits dargelegt, zwingend dem parlamentarischen Gesetzgeber unter Berücksichtigung verfassungsrechtlicher Vorgaben, insbesondere der Grundrechte und Staatszielbestimmungen.

\section{Funktion eines nationalen Landwirtschaftsgesetzes}

4.1 Motive des Gesetzgebers bei Schaffung des geltenden Landwirtschaftsgesetz

Nach langem Drängen des Deutschen Bauernverbandes (DBV) wurde 1955 das Landwirtschaftsgeset $z^{59}$ verabschie- det. ${ }^{60}$ Es erwähnt zwar auch allgemeine Ziele wie die Förderung der Produktivität, sein Kern besteht jedoch darin, der Landwirtschaft ein auskömmliches Einkommen zu sichern - wie es heißt, die ,für sie bestehenden naturbedingten und wirtschaftlichen Nachteile ... auszugleichen" (\$1 LwG). Über die Ursachen der vom DBV damals festgestellten „Einkommensdisparität“ herrschten noch völlig falsche Ansichten, ${ }^{61}$ auch wurden die Preise landwirtschaftlicher Erzeugnisse wie seit über 70 Jahren als Ergebnisse politischer Willensbildung, nicht aber als von Marktkräften bestimmt angesehen. Das Gesetz war voll „Kind seiner Zeit“. $\int 4 \mathrm{LwG}$ fordert einen Vergleich zwischen landwirtschaftlichen und nichtlandwirtschaftlichen Einkommen und dessen Veröffentlichung. Dieser erfolgt sehr einfach: Es wird ein Soll-Einkommen postuliert, bestehend aus einem auBerlandwirtschaftlichen Vergleichslohn, einem Zuschlag für die Betriebsleitertätigkeit und einem Ansatz für die Verzinsung des Eigenkapitals. Diese Summe der Vergleichsansätze wird dem Gewinn aus landwirtschaftlicher Tätigkeit gegenübergestellt und es wird der „Abstand“ zwischen beiden ermittelt. Ist er negativ, verdient die Landwirtschaft zu wenig.

Die Kritik an der Vergleichsrechnung ist so alt wie diese selbst. In einer Serie von Beiträgen hob besonders Schmitt ${ }^{62}$ zahlreiche Mängel hervor und wies darauf hin, dass der Lebensstandard der bäuerlichen Familie und der Gewinn aus dem landwirtschaftlichen Unternehmen zwei verschiedene Dinge sind. Haupterwerbsbetriebe erwirtschaften bis zu 50 Prozent ihres Einkommens aus außerlandwirtschaftlicher Tätigkeit.

Das 66 Jahre alte Gesetz war nie eines zur Regelung des Agrarwesens in seiner Gesamtheit. Der seitherige gewaltige Strukturwandel in der Landwirtschaft würde selbst von einem allein auf Einkommensfragen abzielenden Gesetz andere Inhalte verlangen. Trotzdem gilt die Vergleichsrechnung fort, nach einhelligem Urteil der Wissenschaft „,.. weniger mit ihrer (sehr eingeschränkten) Aussagekraft als vielmehr mit juristischen Verpflichtungen gerechtfertigt". ${ }^{63} \mathrm{Jahr}$ für Jahr wird mit großem Aufwand ein Testbetriebsnetz von etwa 11000 Betrieben ausgewertet, und die Ergebnisse werden in den (freilich nur noch alle vier Jahre erscheinenden) Agrarpolitischen Berichten der Bundesregierung ohne Relativierung veröffentlicht. ${ }^{64}$ Hochrangige Vertreter auf deutscher und europäischer Ebene ${ }^{65}$ sehen in der Vergleichsrechnung nach wie vor den Beweis für einen generellen Einkommensrückstand der Landwirtschaft und eine Rechtfertigung für die Direktzahlungen der „Ersten Säule“.

\subsection{Funktion eines künftigen Landwirtschaftsgesetzes}

Die Funktion eines künftigen Landwirtschaftsgesetzes ist durch die grundgesetzliche Kompetenzordnung be-

57) $\int 5$ Abs. 2 Direktzahlungen-Durchführungsgesetz v. 9.7.2014, BGB1. I S. 897; zuletzt geändert durch Art. 283 der VO v. 19. 6. 2020, BGBl. I S. 1328.

58) $\operatorname{KOM}(2018) 392,393,394$ final.

59) Landwirtschaftsgesetz v. 5. 9. 1955, BGB1. I S. 565

60) Detaillierte Darstellung seiner Entstehung durch einen Mitarbeiter des Münchener ifo-Instituts: Puvogel, Der Weg zum Landwirtschaftsgesetz, 1957.

61) Der Durchbruch kam mit Hanau, Die Stellung der Landwirtschaft in der Sozialen Marktwirtschaft, in: Agrarwirtschaft 7, 1955, S. 1-15.

62) Schmitt, Zur Ermittlung der ,sozialen Lage der in der Landwirtschaft tätigen Menschen" in den Agrarberichten der Bundesregierung. Agrarwirtschaft 33, 1984, S. 301-307; Schmitt, Einkommensermittlung, Einkommensverteilung und -verwendung, Faktorentlohnung und Faktoreinsatz in der Landwirtschaft, Agrarwirtschaft 40, 1992, S. $333 \mathrm{ff}$.

63) Henrichsmeyer/Witzke, Agrarpolitik, 1991, Bd. 1, S. 139.

64) Letztmalig 2019.

65) Busse, NuR 2019, 807, 812f.; Mögele, in: Czybulka/Köck (Hrsg.), Landwirtschaft und Naturschutzrecht, 2019, S. $173 \mathrm{ff}$. 
stimmt, die der Gesetzgeber beachten muss, soweit nicht Grundgesetzänderungen notwendig erscheinen. Dieses Kompetenzmosaik muss kohärenten Zielen zugeordnet sein, um die Regelungen effizient auf diese Ziele auszurichten. Die Abwägung zwischen Ökologie und Ökonomie muss in der Rechtsordnung - zumindest für einen Wirtschaftssektor - einheitlichen Grundentscheidungen folgen, um Wertungskonflikte zu vermeiden. Dies gilt zumindest im horizontalen Verhältnis auf Bundesebene, die sicherlich auf die Landesebene ausstrahlen würde. Das derzeitige Fehlen einheitlicher Grundentscheidungen führt zu inkohärenten, zum Teil widersprechenden Regelungen und Entscheidungen durch die Verwaltungen und die Gerichte, die sich zu Recht mit dieser Aufgabe überfordert sehen. Beispiele hierfür finden sich im Hinblick auf share deals im Grundstückverkehrsrecht, bei der Bewertung des Grünlandumbruchs oder der Futtergrundlage im Rahmen der Tierhaltung.

Diese einheitlichen Grundentscheidungen konkretisieren sich in einem Leitbild, das der Gesetzgeber unter Berücksichtigung der europäischen und föderalen Kompetenzordnung für eine nachhaltige, umweltverträgliche und biodiversitätserhaltende Landwirtschaft entwickeln muss. Dieses Leitbild enthält Entwicklungsvorstellungen und Strategien zu den Betriebsstrukturen und zu den Bewirtschaftungsweisen. Das Leitbild löst insoweit eine verbindliche Beachtenspflicht aus, als bei Nichtbeachtung im Rahmen exekutiver oder judikativer Abwägungsentscheidung ein willkürliches Handeln anzunehmen ist. Der Gesetzgeber muss sich bei der normativen Konkretisierung am Leitbild orientieren, um eine kohärente Regelung zu erzielen. Das Leitbild strahlt jenseits des Umweltrechts auf andere Bereiche aus, die von zentraler Bedeutung für die Landwirtschaft sind: zum einen auf das Agrarzivilrecht wie die Höfeordnung oder das Grundstückverkehrsgesetz, zum anderen auf die Rahmenbedingungen für finanzwirksame Leistungen an die Landwirtschaft.

Ein nationales Landwirtschaftsgesetz würde - dem Beispiel des schweizerischen Landwirtschaftsgesetzes folgend (dort im 3. Titel geregelt) - bei der Ausrichtung und Ausgestaltung der staatlichen und der staatlich geprägten Teile der Unionsbeihilfen in nicht geringem Umfang eine Rolle spielen. Dabei geht es nicht nur darum, was gefördert wird (Umwelt-/Klimamaßnahmen, Tierwohl, demographische Entwicklung), sondern auch in welchem Umfang. Dieser Bedarf nach einer kohärenten Zielsetzung auf der Grundlage eines Leitbilds wird verstärkt durch die Reformansätze der Kommission, ${ }^{66}$ die den Mitgliedstaaten einen weitaus größeren Spielraum bei der Ausgestaltung der Beihilfen einräumen. Dieser Gestaltungsspielraum sollte vom demokratisch unmittelbar legitimierten Gesetzgeber einen Zielrahmen erhalten.

\section{Leitbild und Ziele eines Landwirtschaftsgesetzes}

\subsection{Notwendigkeit und Funktion eines Leitbildes}

Bislang gibt es weder auf nationaler noch auf Unionsebene ein gesetzlich verankertes Leitbild für die Struktur und Funktionen der Landwirtschaft. Die GAP beruht nicht auf einem klar erkennbaren Leitbild, sondern formuliert - unverändert seit den Römischen Verträgen primärrechtlich in Art. 39 AEUV ökonomische Ziele, die sich sowohl auf den Erzeuger als auch auf den Verbraucher beziehen. Sie befassen sich mit der Produktivität der Landwirtschaft, das damit verbundene „Einkommensversprechen" ${ }^{67}$ die Sicherstellung der Versorgung der Verbraucher zu angemessenen Preisen und die Stabilisierung der Märkte. Dieses Defizit der Ziele der GAP kann nur unvollständig durch einen Verweis auf die Querschnittsklauseln Umweltschutz (Art. 11 AEUV) ${ }^{68}$ und Tierschutz (Art. 13 AEUV) aufgefangen werden, die das Zielpro- gramm der GAP ergänzen. Dies gilt ebenso für den erforderlichen Schutz der natürlichen Lebengrundlagen nach Art. 20a GG. ${ }^{69}$

Auf politischer Ebene formuliert die Bundesregierung in ihrem agrarpolitischen Bericht 2019 ein Leitbild einer zukunftsfähigen Agrarpolitik. Danach soll sich „die Politik der Bundesregierung am Leitbild einer nachhaltigen, ökologisch verantwortbaren, ökonomisch leistungsfähigen und regional verankerten Landwirtschaft [orientieren], die einen angemessenen Beitrag zur Erhaltung oder Schaffung attraktiver, lebenswerter und vitaler ländlicher Regionen leistet". ${ }^{70}$ Dieses politische Leitbild könnte als Grundlage für die Entwicklung eines gesetzlichen Leitbildes im Landwirtschaftsgesetz dienen, da es die ökologische, die ökonomische und die regionale Dimension der Landwirtschaft umfasst.

Wie ein politisches Leitbild normativ definiert werden kann, zeigt exemplarisch das schweizerische Landwirtschaftsgesetz von 1998. Nach dessen Art. 1 sorgt ,,der Bund dafür, dass die Landwirtschaft durch eine nachhaltige und auf den Markt ausgerichtete Produktion einen wesentlichen Beitrag leistet zur: a. sicheren Versorgung der Bevölkerung; b. Erhaltung der natürlichen Lebensgrundlagen; c. Pflege der Kulturlandschaft; d. dezentralen Besiedelung des Landes; e. Gewährleistung des Tierwohls“. Art. 2 des schweizerischen Landwirtschaftsgesetzes enthält eine Umsetzungsverpflichtung, ${ }^{71}$ mit der die allgemeinen Kategorien des Art. 1 Landwirtschaftsgesetz ausgefüllt werden. Für die ökologische Dimension relevant sind die verpflichtenden Maßnahmen nach Art. 2 lit. bis des schweizerischen Landwirtschaftsgesetzes. Verpflichtend sind danach Maßnahmen zur Unterstützung einer nachhaltigen Nutzung natürlicher Ressourcen und zur Förderung einer tier- und klimafreundlichen Produktion. Diese Maßnahmen sind erst 2007 eingeführt und 2013 um die tier- und klimafreundliche Produktion erweitert worden.

Der französische Code Rural ${ }^{72}$ verzichtet auf die Entwicklung eines Leitbildes und führt in Art. L1 insgesamt 21 Ziele auf, die die Agrarpolitik Frankreichs prägen sollen. Im Mittelpunkt stehen die ersten beiden Ziele des Art. L1: Versorgungssicherheit der Bevölkerung sowie, damit zusammenhängend, Erhaltung der Leistungs- und Wettbewerbsfähigkeit der Landwirtschaft. Diese prägenden Ziele werden jeweils durch die Vorgabe ergänzt, den Umweltund Landschaftsschutz gleichrangig zu beachten.

Erkennbar wird, dass ein landwirtschaftliches Leitbild so weit offen gestaltet sein muss, um die Vielfalt der Landwirtschaft und ihrer Funktionen abzubilden. Landwirtschaft ist und soll in der Erzeugung (Anbaudiversifizierung, Produktvielfalt, de-intensivierte konventionelle Systeme, Ökolandbau) wie in den Betriebsstrukturen vielfältig sein. Das wird bereits bei den Grundfaktoren deutlich. Schon jetzt gibt es keine einheitliche Landwirtschaft in Deutschland, weder innerhalb des konventionellen Sektors noch auf Landesebene. ${ }^{73}$ Die Betriebsformen, die Bewirtschaftung, aber auch die spezifischen Umweltbeeinträchtigungen sind abhängig von den natürlichen Voraussetzungen sowie von demographischen und marktstrukturellen Begebenheiten.

66) Siehe oben zu 3.4

67) Siehe oben zu 4.1

68) EuGH, Urt. v. 19.9.2002 - C-336/00, Slg. 2002, I-7736, Rdnr. 33.

69) Dazu näher Czybulka, in: Bauer/Czybulka/Kahl/Vosskuhle (Hrsg.) Umwelt, Wirtschaft und Recht, 2002, S. 89-109; Dreier/ Schulze-Fielitz, GG, 3. Aufl. 2015, Art. 20a Rdnr. 44 m.w. N.

70) Agrarpolitischer Bericht der Bundesregierung 2019, S. 12

71) Norer, in: ders. (Hrsg.), Landwirtschaftsgesetz, 2019, Art. 2 Rdnr. 4 LwG.

72) In der Fassung vom 17.9.2020.

73) Bremer, Die rechtliche Gestaltung des Agrarstrukturwandels, 2018, S. $140 \mathrm{ff}$ 
Innerhalb der naturräumlichen Voraussetzungen spielen die Faktoren Klima, Bodenqualität und Relief die größte Rolle.

Das Ziel muss sein, die bestehende natürliche Vielfalt zu erhalten bei gleichzeitiger Lenkung im Hinblick auf die Gemeinwohlziele Biodiversitätserhaltung, Umwelt- und Tierschutz. ${ }^{74} \mathrm{Zu}-$ dem muss die Leistungsfähigkeit der Landwirtschaft auch im Hinblick auf die Erhaltung der Versorgungssicherheit gewährleistet bleiben. Die sich daraus ergebenden konkretisierten Anforderungen lassen sich in diesem Rahmen nur grob skizzieren: Verhinderung von Monokulturen, De-Intensivierung der Landwirtschaft, Stärkung des Ökolandbaus, experimentelle und alternative Betriebsformen. In Erweiterung des Auftrages zur Nahrungs- und Futtermittelerzeugung, muss die Landwirtschaft nach dem Vorbild des schweizerischen Landwirtschaftsgesetzes Aufgaben zur Pflege und Erhaltung der vielfältigen Kulturlandschaft übernehmen. Landwirtschaft muss darüber hinaus möglichst klima- und naturschonend sein, um die Biodiversität der Kultur- und Halbkulturlandschaften zu erhalten und zumeist über entsprechende Förderung - wiederherzustellen, um das Artensterben zu stoppen.

Der ökologische Landbau ${ }^{75}$ ist ein vergleichsweise umweltfreundliches System, von dem auch Innovationsimpulse für die gesamte Landwirtschaft ausgehen. Er sollte deshalb auch weiterhin gefördert werden, auch wenn nachhaltigere Landbausysteme mit höherer Flächennutzungseffizienz als im Ökolandbau denkbar sind. ${ }^{76}$

In ökonomischer Hinsicht muss das Leitbild an einen nachhaltigen Leistungsbegriff anknüpfen, der nicht auf die Erzielung von Höchsterträgen gerichtet ist, weil Höchsterträge häufig zu Lasten der Vielfalt gehen und im Falle der Nutztierhaltung mit dem Tierwohl nicht zu vereinbaren sind. Von daher gehört auch die Regionalisierung der Wertschöpfungsketten zu diesem Leitbild der Landwirtschaft. Damit bezieht sich auch das Ziel, die Ernährungssicherheit der Bevölkerung innerhalb ökologischer Grenzen sicherzustellen, auf den konkreten, vielfältigen „Inhalt“ der im Land erzeugten Produkte und nicht auf abstrakte Umrechnungen (,,ein Bauer ernährt heute 135 Menschen“), während er in Deutschland in Wahrheit an erster Stelle für die Futterherstellung für tierische Erzeugnisse und an zweiter Stelle nachwachsende Rohstoffe für die Energieerzeugung produziert. ${ }^{77}$ Diese regionale Komponente umfasst vielfältige Aspekte der (verloren gegangenen) Kommunikation mit den Landwirten, die sowohl Nahrung als auch „Kollektivgüter" wie blütenreiche Landschaften herstellen. Diese erforderliche Umorientierung ist, wenn sie nicht eine protektionistische Agrarpolitik zugrunde legt, bei gleichzeitigem Erhalt der wirtschaftlichen Anreize für die Landwirte eine schwierige und finanzintensive Aufgabe, die die Gesellschaft herausfordert. Dies zeigt sich derzeit im Bereich der Nutztierhaltung in den Empfehlungen der sog. Borchert-Kommission. ${ }^{78}$

\subsection{Ziele eines Landwirtschaftsgesetzes}

5.2.1 Einbettung in die neue GAP

Das „Neue Umsetzungsmodell“ der künftigen GAP verlangt von den Mitgliedstaaten, ihre Interventionen in einem GAP-Strategieplan darzulegen. Dazu ist ein nationales Landwirtschaftsgesetz in besonderer Weise geeignet, weil es die notwendige Orientierung für die Erarbeitung der Strategie stiftet. Strategiepläne gibt es schon jetzt genug, ${ }^{79}$ aber die erforderliche normative Verankerung fehlte bislang. ,Schaut man sich indes den aktuellen Stand der Umsetzung der Strategiepläne beim BMEL an, so ist schon jetzt festzustellen, dass keine Absicht besteht, durch strukturell neue Wege das Agrarumweltrecht voranzutreiben." 80

Aufgrund dieser europarechtlichen Einbettung sind bei der Erörterung, welche Ziele ein nationales Landwirt- schaftsgesetz verfolgen sollte, auch die Zielvorgaben auf europäischer Ebene in den Blick zu nehmen. Mit der „,neuen GAP" will die Kommission u. a. folgende Ziele verfolgen: ${ }^{81}$ „(e) Förderung der nachhaltigen Entwicklung und der effizienten Bewirtschaftung natürlicher Ressourcen wie Wasser, Böden und Luft; (f) Beitrag zum Schutz der Biodiversität, Verbesserung von Ökosystemleistungen und Erhaltung von Lebensräumen und Landschaften." Ziel f) ist mit dem hier entwickelten Ansatz in vollem Umfang kompatibel, während bei Ziel e) die Terminologie zwar fragwürdig ist, aber offensichtlich auf Art. 11 AEUV (,QQuerschnittsklausel“) angespielt wird und der Umweltschutz einbezogen werden soll.

5.2.2 Ernährungssicherung der Bevölkerung innerhalb ökologischer Grenzen

Art. 5 des Verordnungsentwurfs für den GAP-Strategieplan ${ }^{82}$ definiert als eines der drei Hauptziele der künftigen GAP die „Förderung eines intelligenten, krisenfesten und diversifizierten Agrarsektors, der Ernährungssicherheit gewährleistet". Auf nationaler Ebene finden sich in einzelnen Landesverfassungen Verweise auf die Ernährungssicherheit. ${ }^{83}$ Das Grundgesetz kennt eine Gesetzgebungskompetenz des Bundes zur „Sicherung der Ernährung“ (Art. 74 Nr. 17 GG), ${ }^{84}$ von der aber bislang kein Gebrauch gemacht worden ist.

Gegen den hier vertretenen Vorschlag, die Ernährungssicherung innerhalb der ökologischen Grenzen in den Zielkatalog eines neuen Landwirtschaftsgesetzes aufzunehmen, könnte eingewendet werden, dass jedenfalls die Ernährungssicherung nicht notwendig in ein solches Gesetz gehört, weil schon auf der Unionsebene diese Zielstellung besteht und GAP sowie Binnenmarktpolitik dafür sorgen, dass die Ernährungssicherheit gewährleistet ist. Auch könnte befürchtet werden, dass das Ziel der Ernährungssicherung dazu missbraucht wird, Vorschriften darüber zu erlassen, was in Deutschland anzubauen ist und was nicht. Es liegt uns fern, hierzu Vorschriften zu machen. Wichtig ist uns allerdings die Erhaltung der landwirtschaftlichen Nutzflächen als Ressource der Ernährung, ${ }^{85}$ die Erhaltung der

74) Jurt, Bauern, Biodiversität und ökologischer Ausgleich, 2003, S. 5.

75) Siehe unten zu 5.2.4

76) Ernährungsgutachten, S. XIX. Die EU-Biodiversitätsstrategie sieht für 2030 einen Anteil des ökologischen Landbaus von mindestens 25 Prozent vor.

77) Näher (mit Zahlen) Hampicke, in: Czybulka/Köck (Hrsg.), Landwirtschaft und Naturschutzrecht, 2019, S. 25

78) Empfehlungen des Kompetenznetzwerks Nutztierhaltung vom 11.2.2020, Stand 20.2.2021, abrufbar unter https://www. bmel.de/SharedDocs/Downloads/DE/_Tiere/Nutztiere/ 200211-empfehlung-kompetenznetzwerk-nutztierhaltung.pdf? blob $=$ publicationFile $\& \mathrm{v}=2$

79) Siehe zuletzt die „Ackerbaustrategie 2035“ des BMEL.

80) Martinez, Landwirtschaft und Umweltschutz, in Gesellschaft für Umweltrecht, 43. Fachtagung - Tagungsband 2021, S. 102 (i. E.).

81) Vorschlag der Kommission für eine Verordnung mit Vorschriften für die Unterstützung der von den Mitgliedstaaten im Rahmen der GAP zu erstellenden und durch den Europäischen Garantiefonds für die Landwirtschaft (EGFL) und den Europäischen Landwirtschaftsfonds für die Entwicklung des ländlichen Raums (ELER) zu finanzierenden Strategiepläne (GAP-Strategiepläne) und zur Aufhebung der VO (EU) Nr. 1305/2013 sowie der VO (EU) Nr. 1307/2013 \{SEC(2018) 305 final $\}-\{$ SWD(2018) 301 final\}, S. 13.

82) $\operatorname{COM}(2018) 392$ final

83) Vgl. Art. 55 Abs. 1 Saarländische Verfassung.

84) Seiler, BeckOK, GG, 41. Ed. 15.2.2019, Art. 74 Rdnr. 60. Siehe auch oben zu 2.

85) Hier verbindet sich das Ziel der Ernährungssicherung mit dem allgemeinen Nachhaltigkeitsziel der Flächenverbrauchsbegrenzung für Siedlung und Verkehr. Die Statistiken über die Flächenverbrauchsentwicklung zeigen deutlich, dass das Anwachsen der Siedlungs- und Verkehrsflächen im Wesentlichen zu Lasten der landwirtschaftlichen Nutzflächen geht. 
Landwirtschaft auch und gerade als Träger landbaulicher Erkenntnisse und der Vorrang landwirtschaftlicher Ernährung gegenüber landwirtschaftlicher Energieerzeugung auf den dazu besonders geeigneten landwirtschaftlichen Flächen. Letzteres sollte auch außerhalb von Krisenzeiten dazu führen können, das auf den landwirtschaftlichen Nutzflächen jederzeit wieder zur Herstellung von Nahrungsmitteln zurückgekehrt werden kann.

Dass sich die Ernährungssicherung innerhalb der ökologischen Grenzen zu vollziehen hat, ist ein elementares Gebot der Nachhaltigkeit und ein zentrales Motiv für die Forderung nach einem zukunftsorientierten Landwirtschaftsgesetz. Was Landwirtschaft innerhalb ökologischer Grenzen im Einzelnen bedeutet, wird in den folgenden Abschnitten näher skizziert.

\subsubsection{Umweltgerechte und ressourcenschonende}

\section{Landwirtschaft. ${ }^{86}$}

Die derzeitig praktizierte Landwirtschaft trägt dazu bei, dass wichtige Grenzen überschritten werden, insbesondere durch Bodendegradation, Stickstoffüberschüsse, Biodiversitätsverlusten und Förderung des Klimawandels. Realistischer Weise wird in vielen Regionen Deutschlands eine Verringerung der Umweltbelastungen und eine Wiederherstellung der Biodiversität nur mit einer verringerten Produktion erzielbar sein. Das sowohl im Landwirtschaftsgesetz wie im AEUV (Art. 39 Abs. 1 a) enthaltene Ziel einer Produktivitätssteigerung ist überholt. ${ }^{87}$ Die Produktivitätssteigerung der Landwirtschaft in den letzten Jahrzehnten war so erheblich, dass ,immer mehr“ keinen Sinn mehr ergibt. ${ }^{88}$ Die Spitzen im Ertrag beanspruchen in besonderer Weise Ressourcen und schädigen die Umwelt. Ein typisches Beispiel ist die Düngung. Wird in Erwartung eines Ertrages von neun Tonnen Weizen pro Hektar gedüngt, wirft aber ungünstige Witterung den Ertrag auf sieben Tonnen zurück, so verbleibt ein beträchtlicher Teil des Düngers Schaden verursachend in der Umwelt. Wird von vornherein ein mäßigeres Ertragsziel angestrebt, ist die bedarfsgerechte Steuerung ein geringeres Problem.

Der große Beitrag der Landwirtschaft zum Klimawandel und $\mathrm{zu}$ Umweltbelastungen ist im Wesentlichen bedingt durch die Tierhaltung (und in Verbindung damit der Stickstoffdüngung) und daher letztlich von den Ernährungsmustern der Konsumenten im In- und Ausland abhängig. Eine Abstockung der Tierhaltung in Deutschland würde aber ohne Veränderung der Konsummuster das Problem kurzfristig nur verlagern. Die umwelt- und klimapolitische Bewertung hängt jedoch davon $a b$, ob bei einer Verlagerung der Produktion ins Ausland dort mit geringeren THG-Emissionen produziert werden kann oder nicht. ${ }^{89}$ Ist dies nicht der Fall, besteht die Aufgabe insbesondere der EU darin, den Agrarbinnenmarkt gegenüber nicht nachhaltig produzierten Lebensmitteln außerhalb der EU wirksam zu schützen.

Um zu verhindern, dass Klima- und Umwelt weiter so stark durch die Landwirtschaft betroffen werden, dass die Auswirkungen auf die Ökosysteme und die Gesellschaft unabsehbare negative Folgen entfalten, ist eine drastische Neuausrichtung der Agrarziele zugunsten einer umweltgerechten und ressourcenschonenden Landwirtschaft erforderlich. ${ }^{90}$ Im Mittelpunkt stehen dabei der Schutz von Boden, Wasser und Luft sowie der Erhalt der Produktionsgrundlage. Die in $\$ 1$ Abs. 1 Nr. 2 BNatSchG als Ziel genannte ,nachhaltige Nutzungsfähigkeit der Naturgüter" weist seit jeher in diese Richtung, wird aber (nicht nur) vom Naturschutzrecht bisher nicht ernsthaft verfolgt. Ziel muss eine De-Intensivierung bzw. Extensivierung der landwirtschaftlichen Produktion sein. Weitere Indikatoren für die Umweltverträglichkeit und Ressourcenschonung sind der Grad der Luftverschmutzung, Verlust an Biodiversität, Klimaveränderung, Auswirkungen auf marine
Ökosysteme und Küsten, Beeinflussung der Ozonschicht, Grad der Ressourcennutzung, Verbreitung toxischer Substanzen, Abfallquantität, Wasserverschmutzung und Wasserressource.

Das in $\$ 1$ Nr. 3 PflSchG formulierte Ziel, Gefahren aus der Anwendung von Pflanzenschutzmaßnahmen zu begegnen, wird durch die Grundsätze des integrierten Pflanzenschutzes konkretisiert. Gleichwohl bestehen erhebliche Defizite fort. Sie finden ihre Ursache in den begrenzten sachlichen und personellen Ressourcen der Ämter, um die Auflagen zur Anwendung von Pflanzenschutzmitteln sachgerecht zu überwachen. Zudem muss die Risikobewertung in der Zulassungs- sowie Nachzulassungsphase weiterentwickelt werden und es müssen positive wie auch negative Anreizinstrumente zur effizienten Reduzierung des Einsatzes von Pflanzenschutzmitteln geschaffen werden. ${ }^{91}$

Auch die Reduktion der klimaschädigenden Wirkung der Landwirtschaft muss ein zentrales Ziel des Landwirtschaftsgesetzes sein. Auf die Landwirtschaft einschließlich der THG-Emissionen aus Acker- und Grünlandflächen entfielen $2014^{92}$ mit 104 Millionen Tonnen $\mathrm{CO}_{2}-\ddot{A} q$ rund 11,5 Prozent der Gesamtemissionen Deutschlands (903 Millionen Tonnen $\mathrm{CO}_{2}-\mathrm{A} q$ ). Davon entfielen ca. 42 Millionen Tonnen $\mathrm{CO}_{2}$ aus Ackernutzung von Mooren, Umbruch von Acker- in Grünland und kleinen Quellen. Dies ist schlechte ökologische Praxis und müsste schon deshalb abgestellt werden. Noch immer werden 440000 Hektar Moore ackerbaulich genutzt, was als skandalös zu bewerten ist. Hinzu kommen die erheblichen THG-Emissionen, die bei der Herstellung, Vermarktung und Zubereitung der verzehrten (oder weggeworfenen) Lebensmittel anfallen.

\subsubsection{Bedeutung des Ökolandbaus}

Verbreitet wird die Lösung aller genannten Probleme in einer generellen Umstellung auf ökologischen Landbau gesehen. Er ist umwelt- und naturverträglich und verfolgt u.a.

86) Vgl. den ,allgemeinen Grundsatz“ des $\$ 2$ Abs. 1 Nr. 2 GAK-G, wonach die Erfüllung der Gemeinschaftsaufgabe dazu dient, „die nachhaltige Leistungsfähigkeit ländlicher Gebiete, deren integraler Bestandteil eine umwelt- und ressourcenschonende Land- und Forstwirtschaft ist, zu gewährleisten."

87) So auch Busse, NuR 2019, 807, 809.

88) Hampicke, Wäre in der modernen Landwirtschaft Platz für mehr Artenschutz? Artenschutzreport 44, 2021, S. 24: „Wozu ernten wir neun Tonnen Weizen pro Hektar? Der Bauer weiß es, er braucht das Geld, aber die Gesellschaft? Die neunte Tonne exportieren wir an die Saudis gegen Dollars, die wir nicht brauchen. Auf die achte Tonne könnten wir bei bester leckerer Kost mit nur etwas weniger Fleisch verzichten. Sieben Tonnen Weizen in einem schönen Bestand, am Rand Kornblumen, Klatschmohn und Platterbsen, das würde alle glücklich machen, ließe sich mit geringerem Risiko bedarfsgerecht düngen und ersparte etliche Pestizide."

89) Wissenschaftlicher Beirat für Agrarpolitik, Ernährung und gesundheitlichen Verbraucherschutz, Politik für eine nachhaltigere Ernährung, Juni 2020, S. 208 f.

90) Beispielsweise fallen, um einen Liter Milche zu erzeugen, drei Liter Gülle an, mit den daraus resultierenden Problemen.

91) Hierzu umfassend Nationale Akademie der Wissenschaften (Leopoldina), Der stumme Frühling - Zur Notwendigkeit eines umweltverträglichen Pflanzenschutzes, 2018, S. 12, $24 \mathrm{ff}$.

92) $W B A$ und $W B W$ (Wissenschaftlicher Beirat für Agrarpolitik, Ernährung und gesundheitlicher Verbraucherschutz und wissenschaftlicher Beirat Waldpolitik beim BMEL) 2016: Klimaschutz in der Land- und Forstwirtschaft sowie den nachgelagerten Bereichen Ernährung und Holzverwendung. Gutachten, Berlin, 410 S. zzgl. Anhang. Alle Zahlenangaben aus dem Nationalen Inventurbericht des UBA (in WBA und WBW 2016, S. 19). Die im Klimaschutzprogramm 2030 der Bundesregierung genannten ca. 70 Millionen Tonnen $\mathrm{CO}_{2}$-Äqu. klammern die Moorzerstörung und Grünlandumwandlung aus und „,verrechnen“ sie mit den klimaschützenden Wirkungen des Waldes, so dass die wahren Effekte der Landwirtschaft nicht deutlich werden. 
die Bewahrung und Mehrung der Bodenfruchtbarkeit, die Erzeugung gesunder, insbesondere rückstandsfreier Nahrungsmittel sowie die Förderung des Tierwohls. Die wichtigsten Mittel hierzu sind der Verzicht auf synthetische Pflanzenschutzmittel sowie auf mineralische Düngung im Falle des Stickstoffs vollkommen und beim Phosphor teilweise. Zwar sollte sich der konventionelle Landbau stärker am ökologischen orientieren, z. B. bei der Reduzierung des Pestizideinsatzes. Eine vollständige Übernahme führte aber zu Problemen, die vom Öko-Landbau selbst nirgends angesprochen werden. ${ }^{93}$ Sind eine Mäßigung des Produktionsstresses sowie ein Verzicht auf maximale Erträge und Tierleistungen aus ökologischer Sicht geboten und volkswirtschaftlich ohne Zweifel tolerabel, so sind doch die Flächenerträge im ökologischen Ackerbau so niedrig, dass eine Versorgung der Bevölkerung selbst bei reduziertem Konsum tierischer Nahrungsmittel kaum zu gewährleisten wäre. ${ }^{94}$ In der Öffentlichkeit kursierende Zahlen über Flächenerträge in Öko-Landbau vermitteln ein zu optimistisches Bild. ${ }^{95}$ Hinzu kommt, dass wegen des vollständigen Verbots mineralischer Stickstoff-Düngung 25 bis 30 Prozent des Ackerlandes mit Kleegras oder Luzerne bestellt werden müssten, um genügend Stickstoff in das System einzuschleusen. ${ }^{96}$ Diese große Fläche ginge der Erzeugung pflanzlicher Nahrungsmittel für den Menschen verloren, erzeugte aber eine so große Menge an Futter für Wiederkäuer, insbesondere Milchkühe, dass das Grünland (wie schon jetzt gebietsweise) in die Gefahr des Brachfallens käme, was ökologisch unvertretbar wäre.

Der ökologische Landbau entspricht dem besonders wichtigen Anliegen der Förderung der Biodiversität teilweise und durchaus wirkungsvoll, zum Beispiel im Verzicht auf chemische Herbizide im Ackerbau. Eine lückenlose Zielharmonie derart zu unterstellen, dass im Öko-Landbau alle Anforderungen des Naturschutzes automatisch erfüllt würden, ist aber illusionär. Der Öko-Landbau sieht sich als Hüter und Mehrer der Bodenfruchtbarkeit - Artenvielfalt ist dagegen oft Ergebnis karger, schlecht gedüngter, trockener oder nasser Standortbedingungen, wie sie auch der ÖkoLandwirt ablehnt.

\subsubsection{Tierwohlgerechte Nutztierhaltung}

Die landwirtschaftliche Nutztierhaltung steht im Mittelpunkt der Tierschutzdebatte der letzten Jahre. ${ }^{97}$ Sie findet ihren Ausgangspunkt in erheblichen Defiziten, die unabhängig von der Betriebsform und -größe bereits am Maßstab der derzeitigen Tierschutzstandards festzustellen sind. ${ }^{98}$ Man muss freilich differenzieren: Quälerisch ist die Haltung von Sauen in Käfigen, wo sie sich nicht bewegen können (auch nicht sollen, um nicht ihre Ferkel zu erdrücken). Eine gute Haltungsform sind die derzeit Hunderttausende Mutterkühe mit Kälbern auf der Weide. Tötung durch Schuss und Vermeidung von Lebend-Transporten wären gegenüber den ,üblichen“ Schlachtungs- und Transportmethoden vorzugswürdig. Früher war nicht alles besser, im Gegenteil. Noch von früher herrührende Strukturen sind dem Tierwohl besonders abträglich, wie Anbindeställe für Milchkühe und Mastbullen. Vor 30 Jahren wurden 90 Prozent der Legehennen in Käfigen gehalten, heute etwas über 10 Prozent.

Geprägt wird die Debatte durch einen Wertewandel in der Gesellschaft und in der fachwissenschaftlichen Ethikdiskussion. ${ }^{99}$ Hatten diese Reflexionen zu Tierhaltung und Konsum tierischer Produkte zunächst häufig religiöse Bezüge, treten zunehmend andere gesellschaftliche Strömungen und Weltauffassungen gleichwertig daneben. Eine besondere Bedeutung erhält dabei das kontrakttheoretische Argument des New Deal. ${ }^{100}$ Aus einer soziologischen Perspektive wird die tierschutzrechtliche Debatte auch durch eine gegenseitige Entfremdung der Gesellschaft und der landwirtschaftlichen Produktion geprägt. ${ }^{101}$ Die am modifizierten ethischen Maßstab festge- stellten Defizite lassen erkennen, dass die derzeitigen Haltungsbedingungen eines Großteils der Nutztiere keine Zukunft haben. Sie verlangen nach einer Neuausrichtung am Maßstab von Leitlinien zur Tierhaltung, Tierzucht, Immissionsschutz in der Tierhaltung und tierbezogenem Baurecht. Im Hinblick auf die Besonderheiten des Nutztier-Tierschutzrechts und den agrarstrukturellen Wirkungen wären diese Grundsätze am sinnvollsten auf der Ebene eines gesamtlandwirtschaftlichen Ansatzes im Landwirtschaftsgesetz zu verorten. ${ }^{102}$

Die Anknüpfung allein an die Haltungsbedingungen der Nutztiere greift zu kurz. Ein verminderter Konsum von Fleischprodukten und die Vermeidung von Lebensmittelverlusten und -abfällen würden nicht nur erheblich zum Umwelt- und Klimaschutz im Agrarsektor beitragen, sondern auch die nachteiligen Folgen der Intensivtierhaltung deutlich mindern, wie z.B. die Gewässerbelastung durch Tierarzneimittel und die zunehmenden gesundheitsgefährdenden Antibiotikaresistenzen durch den Antibiotikaeinsatz in der Tierproduktion. ${ }^{103}$

Open Access. Dieser Artikel wird unter der Creative Commons Namensnennung 4.0 International Lizenz veröffentlicht, welche die Nutzung, Vervielfältigung, Bearbeitung, Verbreitung und Wiedergabe in jeglichem Medium und Format erlaubt, sofern Sie den/die ursprünglichen Autor(en) und die Quelle ordnungsgemäß nennen, einen Link zur Creative Commons Lizenz beifügen und angeben, ob Änderungen vorgenommen wurden.

Die in diesem Artikel enthaltenen Bilder und sonstiges Drittmaterial unterliegen ebenfalls der genannten Creative Commons Lizenz, sofern sich aus der Abbildungslegende nichts anderes ergibt. Sofern das betreffende Material nicht unter der genannten Creative Commons Lizenz steht und die betreffende Handlung nicht nach gesetzlichen Vorschriften erlaubt ist, ist für die oben aufgeführten Weiterverwendungen des Materials die Einwilligung des jeweiligen Rechteinhabers einzuholen.

Weitere Details zur Lizenz entnehmen Sie bitte der Lizenzinformation auf http://creativecommons.org/licenses/by/4.0/deed.de.

Open Access funding enabled and organized by Projekt DEAL.

93) Auch nicht in einem über 700-seitigen Konvolut mit hohem wissenschaftlichen Anspruch: Freyer (Hrsg.), Ökologischer Landbau, 2016

94) Berechnung in Hampicke, Kulturlandschaft, 2018, S. 163-164.

95) Oft werden Weizenerträge mit knapp 50 Prozent konventioneller Erträge angenommen. Der Weizen spielt jedoch im ÖkoLandbau eine geringere Rolle, dafür sind (ökologisch zu begrüBen) ertragsschwache Kulturen wie Dinkel und Hafer stärker vertreten.

96) Nur Kulturpflanzen aus der Familie der Fabaceae (Leguminosen) leben in Symbiose mit Knöllchenbakterien, die mittels des Enzyms Nitrogenase Stickstoff aus der Luft fixieren können.

97) Martinez, in: Rechtswissenschaft 3/2016, S. 441-467. Graef, NuR 2020, 604ff.

98) Siehe hierzu umfassend das Gutachten des Wissenschaftlichen Beirats für Agrarpolitik, Wege zu einer gesellschaftlich akzeptierten Nutztierhaltung, 2015

99) Martinez, in: Rechtswissenschaft 3/2016, S. 450f.; Discherl, Fleischkonsum und Tierhaltung in der aktuellen gesellschaftsethischen Debatte, Berichte über Landwirtschaft 91, 2013, S. 1; Grimm, in: Grimm/Otterstedt (Hrsg.): Das Tier an sich. Disziplinenübergreifende Perspektiven für neue Wege im wissenschaftsbasierten Tierschutz, 2012, S. 276-296.

100) Zühlsdorf/Kühl/Gauly/Spiller, Wie wichtig ist Verbrauchern das Thema Tierschutz? Präferenzen, Verantwortlichkeiten, Handlungskompetenzen und Politikoptionen - Kommentiertes Chart-book zur repräsentativen Umfrage, 2016; S. 16. Siehe ferner die Beiträge in: Dawkins/Bonney (Hrsg.), The Future of Animal Farming - Renewing the Ancient Contract, 2008.

101) Kayser/Böhm/Spiller, in: Yearbook of Socioeconomics in Agriculture 2011, S. 59-83.

102) Siehe Teil 2 zu 2.5.

103) Dazu näher Köck, ZUR 2020, 464, 468 f. 